\title{
OBSERVATIONAL RESULT ON X-RAYS
}

by Philip C. Fisher, Dwight B. Clark, Arthur J. MeYerott and Kermit L. Smith

(Lockheed missiles and Space Company

Research Laboratories

Palo Alto, California, U. S. A.)

Résumé. - On donne les résultats finaux de la recherche de sources de rayons $X$ mous dans le ciel nocturne (20 mars 1963). Une seule région du Ciel a fourni un signal supérieur à 2,5 à 3 fois l'écart statistique le plus probable alors que la probabilité d'observer un écart statistique fortuit de cet ordre est de 0,2 . La région est située à $\alpha=23 h 40 m, \delta=+78^{\circ}$. Il ne semble pas y avoir de variations $d u$ fond galactique émettant des rayons $X$ mous avec la latitude galactique et ce résultat est discuté.

SUMMARY. - Final results are presented for a March 20, 1963 search for night sky sources of soft X-rays. Although one sky region was found to be associated with an X-ray flux 2,5 to 3 standard deviations above background, the probability of observing one random fluctuation of this importance in the data for the night sky is estimated to be as high as $20 \%$. The region is located at $\alpha=23 h 40 \mathrm{~m}, \delta=+78^{\circ}$. Apparently negative results from a search of the data for variations of $X$-ray background with galactic latitude are presented and discussed.

Резюме. - Даны окончательные результаты расследования источников мягких лучей $\mathrm{X}$ в ночном небе (20 марта 1963). Только один участок неба дал сигнал превышающий в 2,5-3 раза наиболее вероятное статистическое отклонение, тогда как вероятность наблюдать случайное статистическое отклонение этого порядка равна 0,2. Участок определен координатами $\alpha 23=40$ м $\delta=+78^{\circ}$. Как будто нет изменений галактического фона, излучающего мягкие лучи $\mathrm{X}$, с галактической широтой и этот результат обсужден.

\section{InTRODUCTION.}

On March 20, 1963, a rocket experiment was performed in an attempt to locate and obtain spectral information about stellar sources of soft $\mathrm{X}$-rays in the $0.2-20 \mathrm{keV}(60-0.6 \AA)$ range. The X-rays were detected by several weil collimated $\left(5^{\circ} \times 10^{\circ}\right)$ gas filled proportional counters protected by anticoincidence plastic-scintillatorphotomultiplier shields. Preliminary results of this Aerobee 4.70 rocket flight and an earlier similar experiment on Aerobee 4.69 have already been presented [3]. Final results for the Aerobee 4.70 flight are given here.

\section{ISOPHOTE PLOT CONSTRUCTION.}

Figure 1 is a celestial sphere isophote plot derived from all counts recorded by the Aerobee 4.70 detectors and is based on a more accurate and extended vehicle aspect solution than was used for the preliminary results [3]. The extended solution permitted inclusion of data from two more scans (to a total of 11 scans) of the sky.
Model atmosphere calculations have shown the flux of night sky X-rays from the two additional scans are essentially free of absorption effects. The rocket's $0.5-3.5 \mathrm{keV}$ and $2-20 \mathrm{keV}$ counters alternately observed the upward (night) sky and the downward (earth-obscured) sky. Each detected event was assigned to an elemental area of the celestial sphere. 'The rocket's motion was such that individual elemental areas were scanned from 2 to 9 times by each of the detectors. Observed events were also segregated into two broad and overlapping energy intervals, $0.5-8 \mathrm{keV}$. and $4-20 \mathrm{keV}$. Preliminary results from this revised data analysis procedure were presented at The Meeting on Neutron Stars and Celestial X-Ray Sources held in New York on March 20, 1964. A noticeably high count rate in a given sphere region was compared to the background count-rate predicted for that particular region in order to assess the statistical significance (number of standard deviations $\sigma$ above background) of the high count rate. Although $\sigma$ values for the night and earth-obscured sky were based on slightly different (and independently calculated) back- 
ground rates, for each sky region all detected events were assumed to occur randomly in time. Because both the night and earth-obscured sky were arbitrarily terminated $15^{\circ}$ from the horizontal horizon, the night sky background was used for the horizon region.

An allowance for the variation of detector efficiency with angle off the counter's centerline was made as described in [3]. After the isophote plot was finished, the $\sigma$ value for each high count region was recomputed using the total of all possible counts (no allowance for angular distribution) which might be associated with the high count location. While the differently computed $\sigma$ values of a given high count region never differed by more than $0.5 \sigma$, all Figure 1 regions are shown with the lowest $\sigma$ values computed.

\section{Results.}

With the exception of what appear to be horizon related sources, only a single region is as much as $2.5 \sigma$ above the predicted background. The count rate was noticeably high on five or the seven successive passes over this region and the actual counts in the $0.5-8 \mathrm{keV}$ interval total 25 as compared to a predicted background of 14 . Unfortunately, the relatively stronger Scorpius source $[1,5,6,7]$ was below the rocket horizon on both the Aerobee 4.69 and 4.70 flights and so could not have been observed. Our failure to detect the Crab Nebula source observed by the Naval Research Laboratory group has been particularly noted by BowYER [2] and several of his remarks have been discussed in a Letter by Fisher and Meyerott [4]. Figure 1 contains data from 3 scans directly over Ml, as compared to the single direct scan (out of a total of 3 scans) utilized in [3]. However, the total $0.5-8 \mathrm{keV}$ interval count for the Crab Nebula location of 9 (compared to a background of 11) is still statistically less significant than the count for several other regions scanned. Either our : $a$ ) low detection sensitivity ( 1/5 that on the NRL flight) or $b$ ) fairly broad energy intervals (which effectively suppress sensitivity), may be the cause of our not detecting the Crab Nebula source.

Several investigations of the correlation between high count regions and approximate celestial sphere position have been made in addition
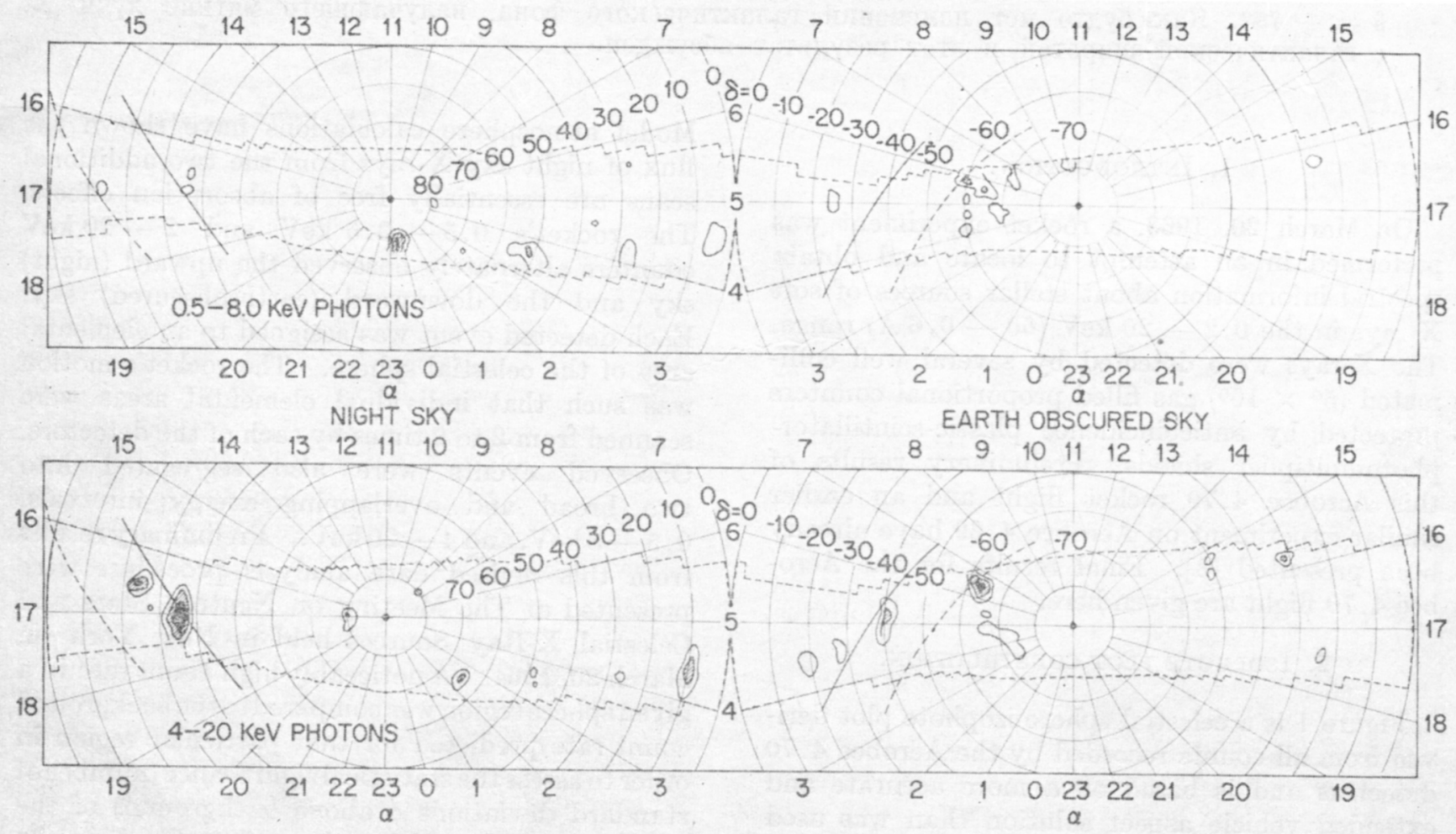

Fra. 1. - Celestial sphere isophote plots of high count regions observed by Aerobee 4.70 detectors. The solid and dashed lines separa. ting the night sky from the earth obscured sky represent the horizontal and rocket horizons respectively. The simply outlined, cross-hatched, and blackened areas indicate count rates $1.5 \sigma, 2.0 \sigma$ and $2.5 \sigma$ above background. 
to the search for point source locations discussed above. Figure 2 contains the results of a correlation attempt in which the area of Figure 1 high count regions (in terms of square degrees) was plotted along the line down the center of the combi-

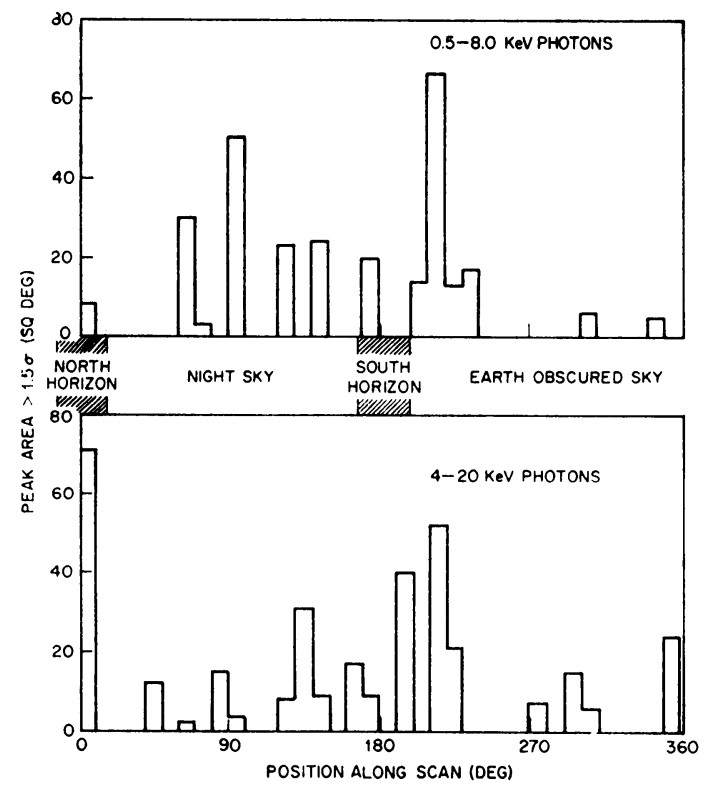

Fig. 2. - Test of Fig. 1 results for clumping of high count regions along the line of scan.

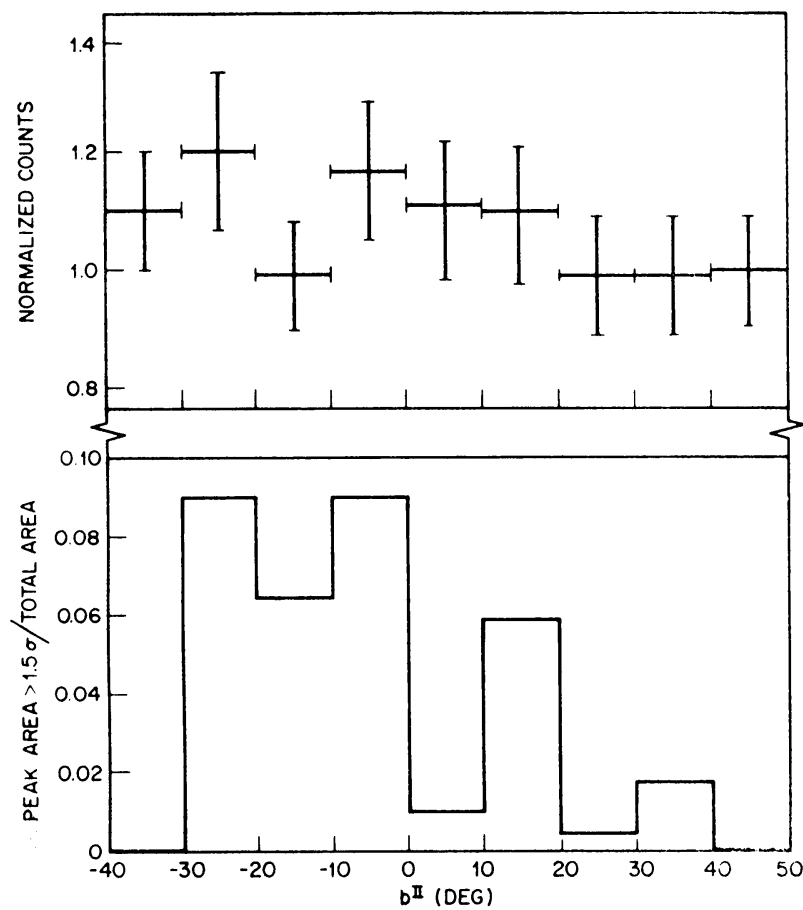

Fra. 3. - Plots of 0.5-8.0 keV X-ray data for evaluating galactic latitude dependence. The top portion of the figure presents all counts observed, while the bottom portion of the figure represents only the counts $1.5 \sigma$ above background. ned scans given in Figure 1. The only finding of apparent significance is the previously mentioned clumping of high count regions near the earth's horizons.

Figure 3 gives the results of two other correlations attempted, both of these particular correlations being based on data for the $0.5-8 \mathrm{keV}$ energy range. Normalization for the top portion of Figure 3 was derived by comparison with the average value of $1.88 \pm .07$ counts $/ \mathrm{cm}^{2}$-s obtajned from a similar plot for the earth-obscured sky. The apparent $6 \%$ excess of $0.5-8 \mathrm{keV}$ night sky (over earth-obscured sky) photons actually amounts to $.11 \pm .07$ counts $/ \mathrm{cm}^{2}-\mathrm{s}$ and is almost completely due to $2-8 \mathrm{keV}$ photons. After allowing for the $1 / 50 \mathrm{Sr}$ effective solid angle of the collimator and the 0.7 value of counter efficiency, the excess counting rate is found to be equivalent to $8 \pm 5$ photons $/ \mathrm{cm}^{2}$-s-sr. Previous measurements of this quantity $[1,7]$, yielded a value of 6 photons $/ \mathrm{cm}^{2}$-s-sr. Similar treatment of the $4-20 \mathrm{keV}$ data shows more counts per second trom the earthobscured sky than from the night sky. Consequently the $\mathrm{X}$-ray flux from the night sky is indeed somewhat softer than that observed when looking down at the earth's atmosphere.

Results of a third correlation attempt are given in the bottom portion of Figure 3. The area of Figure 1 high count regions (in terms of square degrees, using $\sigma$ values derived from the angularly distributed counts) was examined for a galactic latitude dependence. A trivial correction was made to allow for the fact that the rocket's precession resulted in slightly different degrees of overlap of the many successive scans over each latitude interval. Although a latitude variation does appear, the actual $\mathrm{X}$-ray fluxes are so small as to render the variation of questionable statistical significance. Except for one peak in the $-20^{\circ}$ to $-30^{\circ}$ latitude interval, a similar investigation of the counts associated with $4-20 \mathrm{keV}$ pulses shows no latitude variation.

\section{Conclusions.}

Complete re-analysis of the Aerobee 4.70 flight, utilizing all data available, has led to approximately the same quantity and location of high count regions given previously [3]. However, because of their low statistical significance, the Aerobee 4.70 data alone are insufficient to establish the real existence of any of the high count regions. The two possible exceptions to this finding are the aggregate of regions in the vicinity of the 
earth's horizon and the region at $\alpha=23^{h} 40^{\mathrm{m}}$, $\sigma=+78^{\circ}$. A night sky flux of $8 \pm 5$ photons/ $\mathrm{cm}^{2}-\mathrm{s}-\mathrm{sr}$ found for the 4-8 $\mathrm{keV}$ range agrees well with values obtained by other observers. The 8-20 keV X-ray flux from the earth's atmosphere is noticeably larger than that from the night sky. A variation (of uncertain statistical significance) with galactic latitude was found for the
0.5-8 keV X-rays coming from a swath of sky which cuts diagonally across the galactic equator near $160^{\circ}$ of longitude. This work was supported by the National Aeronautics and Space Administration under contract NAS 5-1174 and by the Lockheed Independent Research Program.

Manuscrit reçu le 18 septembre 1964.

\section{REFERENCES}

[1] Bowyer S., Byram E. T., Chubb T. A. and Fritedman H., 1964, Nature, 201, 1307.

[2] BowYer S., 1964, Ap. J. 140, 820.

[3] Fisher P. C. and Meyerott A. J., 1964, $A p$. J., $189,123$.

[4] Fisher P. C. and Meyerott A. J., 1964, Ap.J. 140,821 .
[5] Giacconi R., Gursky H., Paolini F. R. and Rossi B. B., 1962, Phys. Rev. Letters, 9, 439.

[6] Giacconi R, Gursisy H., Paolini F. R. and Rossi B. B., Space Research, V, p. 831.

[7] Gursky H., Giacconi R., Paolini F. R. and Rossi B. B., 1963, Phys. Rev. Letters, 11, 530. 